\title{
Comparison of two types of fixation for proximal tibial epiphysiodesis: An experimental study in a rabbit model
}

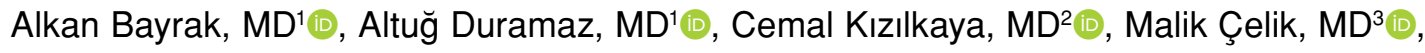

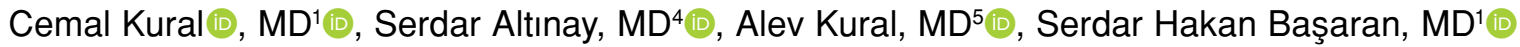 \\ 'Department of Orthopedics and Traumatology, University of Health Sciences, Bakırköy Dr. Sadi Konuk Training and Research Hospital, Istanbul, Turkey \\ ${ }^{2}$ Department of Orthopedics and Traumatology, Bahçelievler State Hospital, Istanbul, Turkey \\ ${ }^{3}$ Department of Orthopedics and Traumatology, Batman State Hospital, Batman, Turkey \\ ${ }^{4}$ Department of Pathology, University of Health Sciences, Bakırköy Dr. Sadi Konuk Training and Research Hospital, Istanbul, Turkey \\ ${ }^{5}$ Department of Biochemistry, University of Health Sciences, Bakırköy Dr. Sadi Konuk Education and Research Hospital, Istanbul, Turkey
}

Growth plate or the physis is a cartilage structure that occurs at the distal part of the long bones and provides bone elongation. Physeal injuries are difficult to treat and cause complications such as growth arrest, progressive angular deformities, and limb length discrepancy after childhood physeal damage. ${ }^{[1]}$ After traumatic epiphyseal injuries, a physeal bar may occur that may lead to progressive angular deformities or limb shortness during the fracture healing. ${ }^{[1,2]}$ Various hemiepiphysiodesis techniques have been developed for preventing deformity. ${ }^{[3,4]}$ Blount ${ }^{[5]}$ showed that the growth disturbances such as angular deformities can be controlled by using minimally invasive hemiepiphysiodesis technique with $U$ nails. Since $U$ nail migration and breakage

Received: November 26, 2020

Accepted: March 03, 2021

Published online: June 11, 2021

Correspondence: Alkan Bayrak, MD. SBÜ Dr. Sadi Konuk Eğitim ve Araştırma Hastanesi Ortopedi ve Travmatoloji Kliniği,

34147 Bakırköy, İstanbul, Türkiye.

E-mail: drqueum@gmail.com

Doi: $10.52312 /$ jdrs.2021.80219

Citation: Bayrak A, Duramaz A, KIzllkaya C, Celik M, Kural C, Altınay $S$, et al. Comparison of two types of fixation for proximal tibial epiphysiodesis: An experimental study in a rabbit model. Jt Dis Relat Surg 2021;32(2):468-477.

(02021 All right reserved by the Turkish Joint Diseases Foundation

This is an open access article under the terms of the Creative Commons Attribution-NonCommercial License, which permits use, distribution and reproduction in any medium, provided the original work is properly cited and is not used for commercial purposes (http://creativecommons.org/licenses/by-nc/4.0/).

\section{ABSTRACT}

Objectives: In this study, we describe a novel hemiepiphysiodesis technique to prevent implant-related perichondrial ring injury in a rabbit model.

Materials and methods: Proximal tibial epiphyseal plates of a total of 16 white New Zealand rabbits were used for this animal model. The subjects were divided into three equal groups as follows: Group 1 (Kirschner wire [K-wire]/cerclage), Group 2 (8-plate) right-hind legs, Group 3 (Control) left hind legs. Using anteroposterior radiography, the medial slope angle (MSA), articular line-diaphyseal angle (ALDA), and the angle between screws of 8-plate in lateral X-ray tibial slope angle (TSA) were measured. The radiographs were taken early postoperative (Day 1) and on sacrification day (Week 8). The histological evaluation of the perichondrial ring was made on a 7-mm axial section that stained with Safranin O/fast green at $\times 10$ magnification.

Results: In both K-wire and 8-plate groups, the early postoperative ALDA and TSA were greater than the sacrification ALDA and TSA ( $\mathrm{p}=0.028$ and $\mathrm{p}<0.001$, respectively). The early postoperative MSA was lower than the sacrification MSA in groups, $(\mathrm{p}<0.001)$. The MSA in the control group was lower than the K-wire and 8-plate groups $(\mathrm{p}<0.001$ and $\mathrm{p}=0.009$; respectively). The perichondrial ring thickness of the K-wire group was greater than the 8-plate group in histological evaluation $(\mathrm{p}<0.001)$.

Conclusion: Both of the K-wire and 8-plate groups showed similar angulation effects in the proximal tibia, although histologically less damage to the perichondrial ring was observed in the $\mathrm{K}$-wire group, compared to the 8-plate group.

Keywords: Eight plate, hemiepiphysiodesis, histology, K-wire, perichondrial ring.

may occur, the tension band hemiepiphysiodesis technique by 8-plate and screws is currently preferred in the treatment of childhood deformities. ${ }^{[1,3,6]}$ These procedures temporarily inhibit limb growth. However, in studies evaluating U-nails and 8-plates, the development of permanent epiphysiodesis has 
been reported associated with periosteal damage during implant application or removal. ${ }^{[7,8]}$ Aykut et al. ${ }^{[7]}$ emphasized that a bar was formed due to damage to the perichondral ring in the proximal tibial epiphysiodesis of the rabbit model by using $U$ nail and that U nail should be extraperiostally performed.

The 8-plate provides epiphysiodesis successfully at rates ranging from 74 to $91 \% . .^{[9,10]}$ However, some patients undergoing epiphysiodesis with 8-plate experience problems, such as permanent epiphysiodesis and implant failure. ${ }^{[11,12]}$ This problem can be caused by damage to the perichondrial ring around the physis. ${ }^{[9,13]}$ In the literature, it has been shown in various animal experiments that 8-plate can damage the perichondrial ring and cause permanent damage in this area. ${ }^{[9,14]}$ Tension band technique with Kirschner wire (K-wire) and cerclage are frequently used in the fixation of the olecranon and medial malleolar fractures. The tension band fixation provides an advantage for fracture union by converting tensile forces into compressive forces. ${ }^{[15,16]}$ In the present experimental animal study, we hypothesized that the surface where the tension band application with the K-wire creates pressure on the perichondrial ring would be lower than the 8-plate and K-wire would cause less damage to the perichondrial ring. We, therefore, aimed to develop a lower-cost technique that prevents angular deformities, does not cause permanent problems, and minimizes damage by reducing the pressure in the perichondrial ring, by using the tension band technique with a K-wire and cerclage, which are commonly used by orthopedic surgeons in clinical practice.

\section{MATERIALS AND METHODS}

In this experimental study, a total of 16 , six-week-old New Zealand rabbits weighing between 750 and $1,000 \mathrm{~g}$ were used. The study protocol was approved by the Bağcılar Training and Research Hospital Experimental Research and Skill Development Center (IRB approval ID: 2017/33). All animals involved in the experiment received humane care in compliance with the Guide for the Care and Use of Laboratory Animals (www.nap.edu/ catalog/5140.html). Ketamine and xylazine were used for anesthesia, and meperidine was used for postoperative analgesia. The proximal physis of the subjects was studied on the tibia of the right hind legs as Group $1(\mathrm{n}=8)$ and Group $2(\mathrm{n}=8)$, and randomly selected left hind legs of Groups 1 and 2 were studied as Group $3(n=8)$. The subjects were randomly divided into three groups as follows:
Group 1 (K-wire and cerclage group): Eight rabbit right hind legs, the tension band technique was applied using K-wire and cerclage.

Group 2 (8-plate group): Eight rabbit right hind legs, the tension band technique was applied using 8-plate and screws.

Group 3 (Control group): The untreated eight left hind legs were randomly selected of the subjects in Groups 1 and 2 which were sacrificed formed the control group as Group 3. All rabbits followed for eight weeks.

\section{Anesthesia, premedication, and surgical procedure}

Surgical procedures were performed under general anesthesia. A dose of $35 \mathrm{mg} / \mathrm{kg}$ ketamine (10\% injectable Alfamine ${ }^{\circledR}$; Alfasan International BV, Woerden, the Netherlands) and a dose of $5 \mathrm{mg} / \mathrm{kg}$ xylazine (Ksilazol ${ }^{\circledR}$; Provet, Istanbul, Turkey) were mixed and intraperitoneally administrated at a dose of $0.4 \mathrm{~mL} / \mathrm{kg}{ }^{\left[{ }^{[7]}\right.}$ After anesthesia was provided, the right leg was prepared sterile by staining with a solution of polyvinylpyrrolidone-iodine complex (Batticon $^{\circledR}$; Adeka Corp., Istanbul, Turkey). A 2 to 3-cm anteromedial longitudinal incision, slightly medial to the tibial tubercle, was performed in the proximal of the right tibia. Soft tissues such as subcutaneous tissue and joint capsule were dissected and the medial collateral ligament distal insertion was identified and served as a landmark. As a white line, the physis was easily distinguished from the surrounding bone. The joint surface was reached and one K-wire was placed as a guide parallel to the joint surface. In parallel with this guide, two 1-mm K-wires were applied in parallel to the proximal joint of the tibia via the epiphyseal plate. The distance between the K-wires was $5 \mathrm{~mm}$ in the sagittal plane. The hole for cerclage wire opened in the middle of the tibia in the sagittal plane by a $1.2-\mathrm{mm}$ drill. Then, 1-mm cerclage wire was passed through the hole opened 1 to $2 \mathrm{~cm}$ distal to the epiphyseal plate and tightened simultaneously until proximally admitted K-wires bending slightly. The K-wires were cut by twisting at a close distance from the periosteum and embedded under the skin (Figure 1). After washing with saline, the fascia and skin were closed with $3 / 0$ Vicryl and $2 / 0$ prolene sutures, respectively. The 8-plates (Guided Growth System; Orthofix Corp., Verona, Italy) were performed in Group 2 after placing one K-wire as a guide parallel to the joint surface with the same surgical approach. The 8-plate was applied extraperiosteally using one proximal- and one distal screw (Figure 1). After washing with saline, the fascia and skin were closed with $3 / 0$ Vicryl and 2/0 prolene sutures, respectively. 
After the wound closure in the laboratory, standard anteroposterior and lateral X-rays were taken by portable X-ray fluorescence and the position of the surgical devices was controlled. In the postoperative period, ceftriaxone was administered intramuscularly at the dose of $50 \mathrm{mg} / \mathrm{kg}$ every $12 \mathrm{~h}$ for prophylaxis for $24 \mathrm{~h}$. All subjects were returned to normal cage activities. ${ }^{[1]}$ Meperidine was administered at a dose of $6 \mathrm{mg} / \mathrm{kg}$ every 4 to $6 \mathrm{~h}$ for analgesia for three days.

\section{Radiographic measurements and histological evaluation}

The subjects were evaluated on the first postoperative day and eighth week during the sacrification phase with two plain radiographs.
A portable X-ray fluorescence was used for the graphs. The articular line-diaphyseal angle (ALDA; the angle between the tibial anatomical axis and the tibial proximal joint line) and the medial slop angle (MSA; the angle between the tibial anatomical axis and the medial plateau joint line) were evaluated using anteroposterior radiographs (Figures 2 and 3). ${ }^{[7,9]}$ The tibial slope angle (TSA; the angle between the tibial anatomical axis and the tibial joint line) was evaluated using lateral radiographs. The angles between the screws in the 8-plate group were also measured by anteroposterior radiographs..$^{[9]}$ The RadiAnt DICOM 2.2.9 Viewer (Medixant, Poznan-Poland) program was used for measurements of all three groups
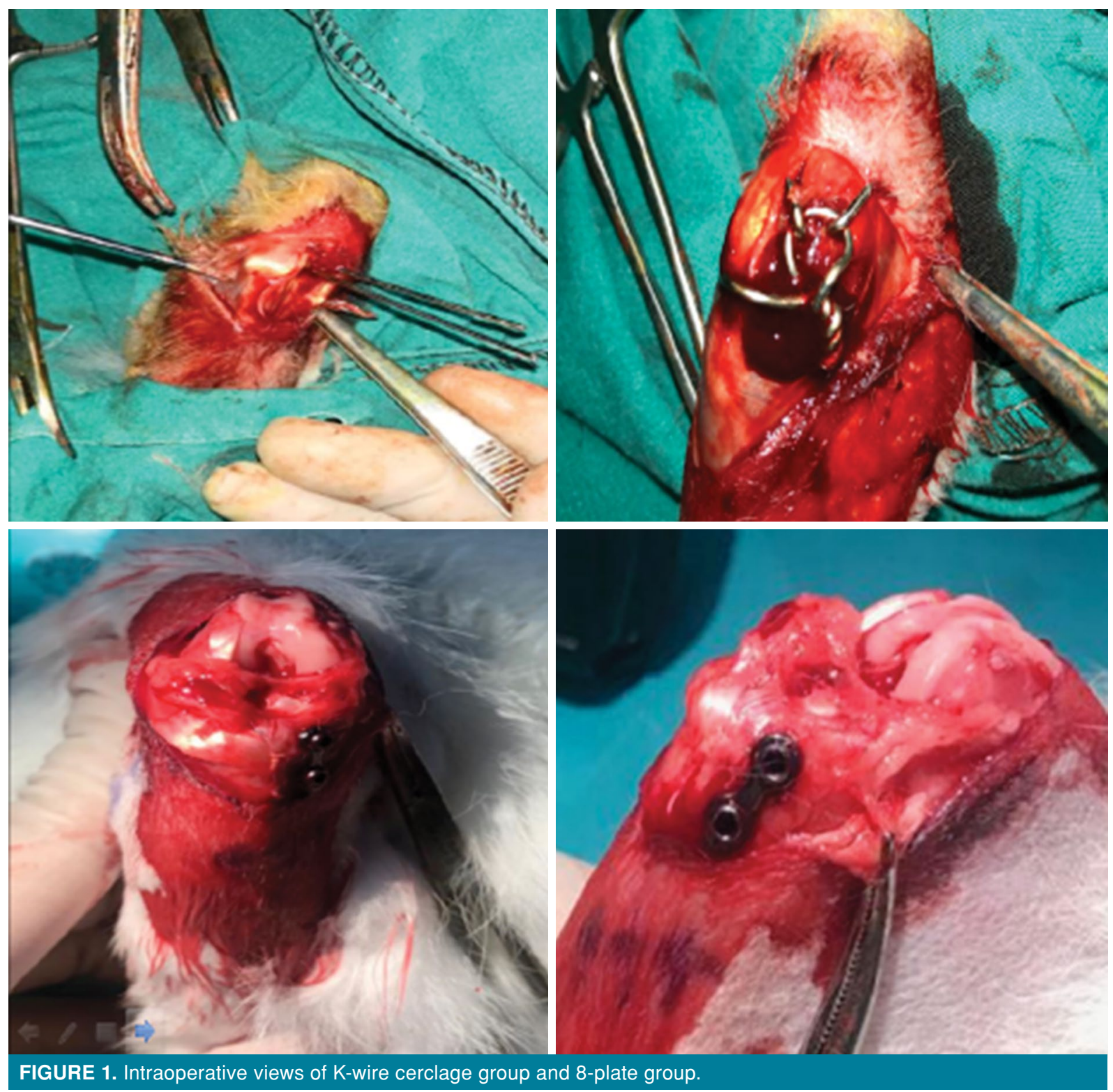
(K-wire, 8-plate, and control). ${ }^{[17]}$ After eight weeks, the subjects were sacrificed and the right tibias of Group 1 and right tibias of Group 2 were taken. Eight left tibias of the subjects in Group 1 and 2 were randomly used for the control group. The proximal one third of the tibia was separated, the implants were removed and decalcified by depositing in a solution containing $10 \%$ formaldehyde. All samples were taken from the affected side of the perichondrial ring, such as the contact area with the plate and the cerclage. Totally, 7-mm axial sections were taken from the samples and stained with Safranin-O/fast green. As the perichondrial ring and growth plate is not uniform, its width was studied in different samples. To minimize errors (i.e., the evaluation of the unaffected area), three-section measurements from samples taken from each subject were measured and averaged. ${ }^{[18]}$ The thickness of the perichondrial ring was evaluated at $\times 10$ magnification and compared between groups (Figure 4). The same pathologist
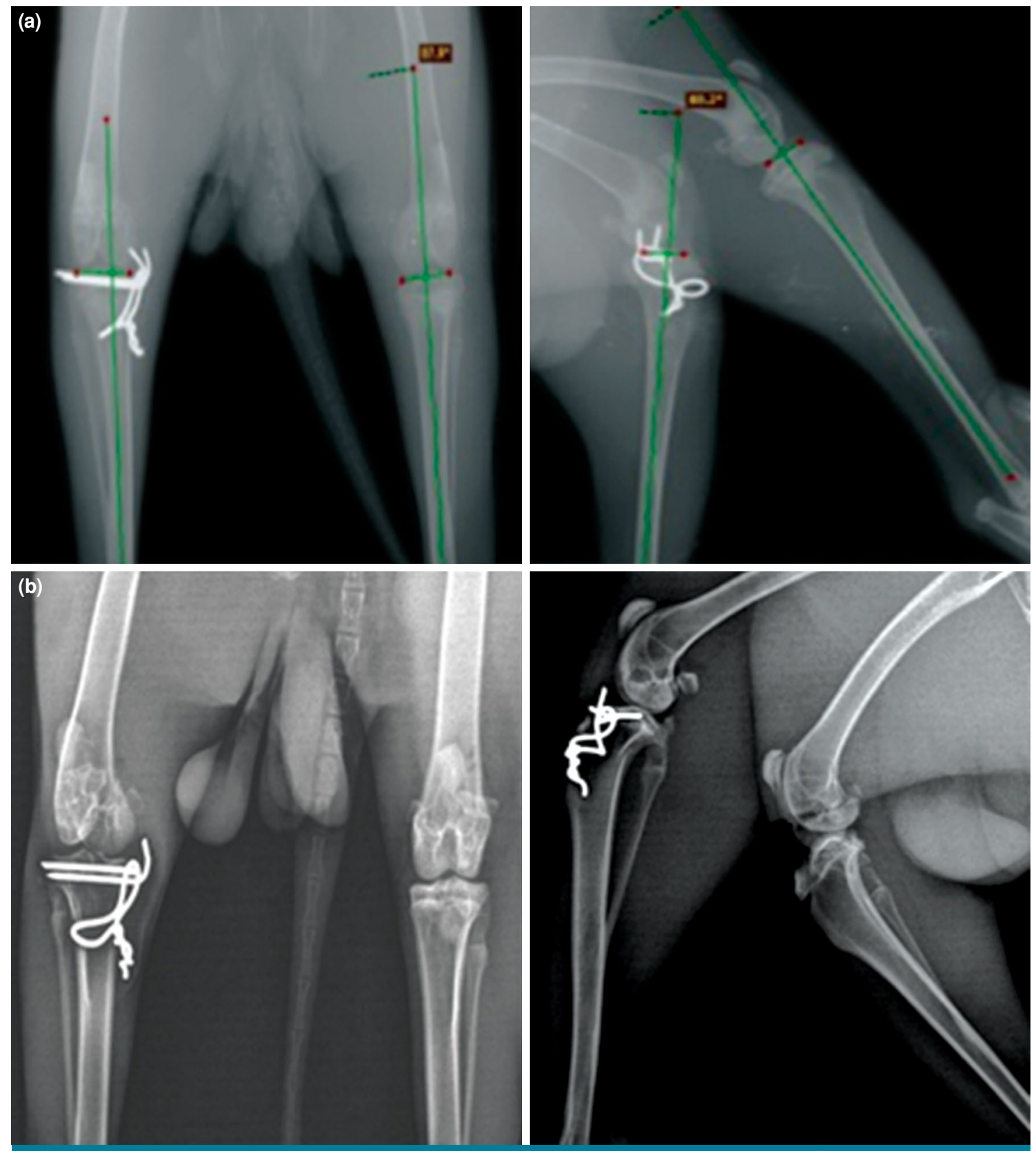

FIGURE 2. (a) Early postoperative (Day 1) anteroposterior and lateral radiographs and (b) $8^{\text {th }}$-week anteroposterior and lateral radiographs of the fixation of the subject undergoing epiphysiodesis using K-wire and cerclage. 
who was unaware of the group allocation prepared and evaluated the samples in a blinded manner. The cumulative cost of the implants used in Group 1 and Group 2 was calculated in each group and was compared between groups.

\section{Statistical analysis}

Statistical analysis was performed using the Number Cruncher Statistical System (NCSS) version
2007 software (NCSS LLC, Kaysville, UT, USA). Descriptive data were expressed in median (first quarter to third quarter). The suitability of quantitative data for normal distribution was tested using the Shapiro-Wilk test and graphical examinations. The Mann-Whitney $U$ test was used to compare the quantitative variables that did not show normal distribution between the two groups. The KruskalWallis test and Dunn-Bonferroni test were used for
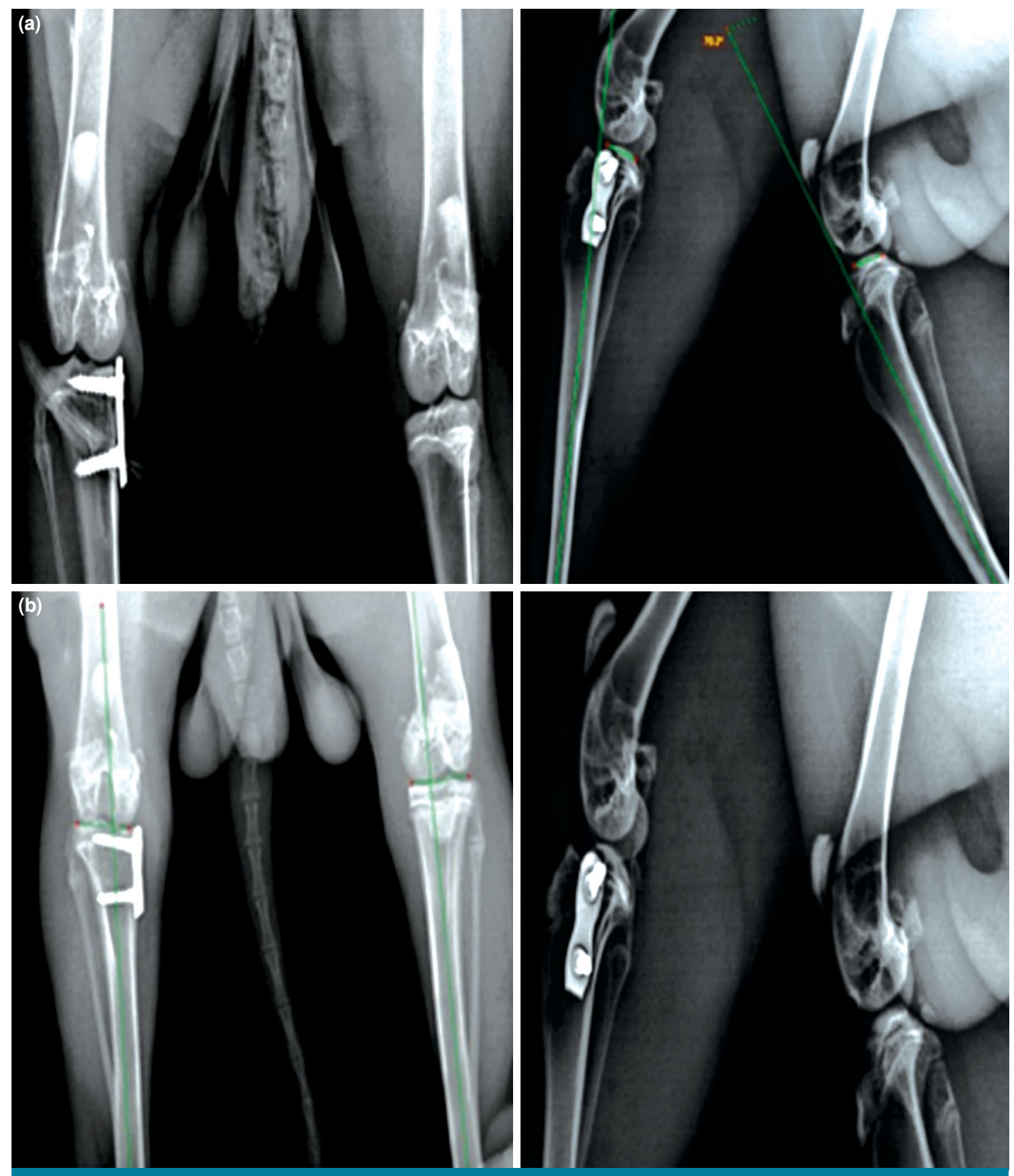

FIGURE 3. (a) Early postoperative (Day 1) anteroposterior and lateral radiographs and (b) $8^{\text {th }}$-week anteroposterior and lateral radiographs of the fixation of the subject undergoing epiphysiodesis using 8-plate and screws. 

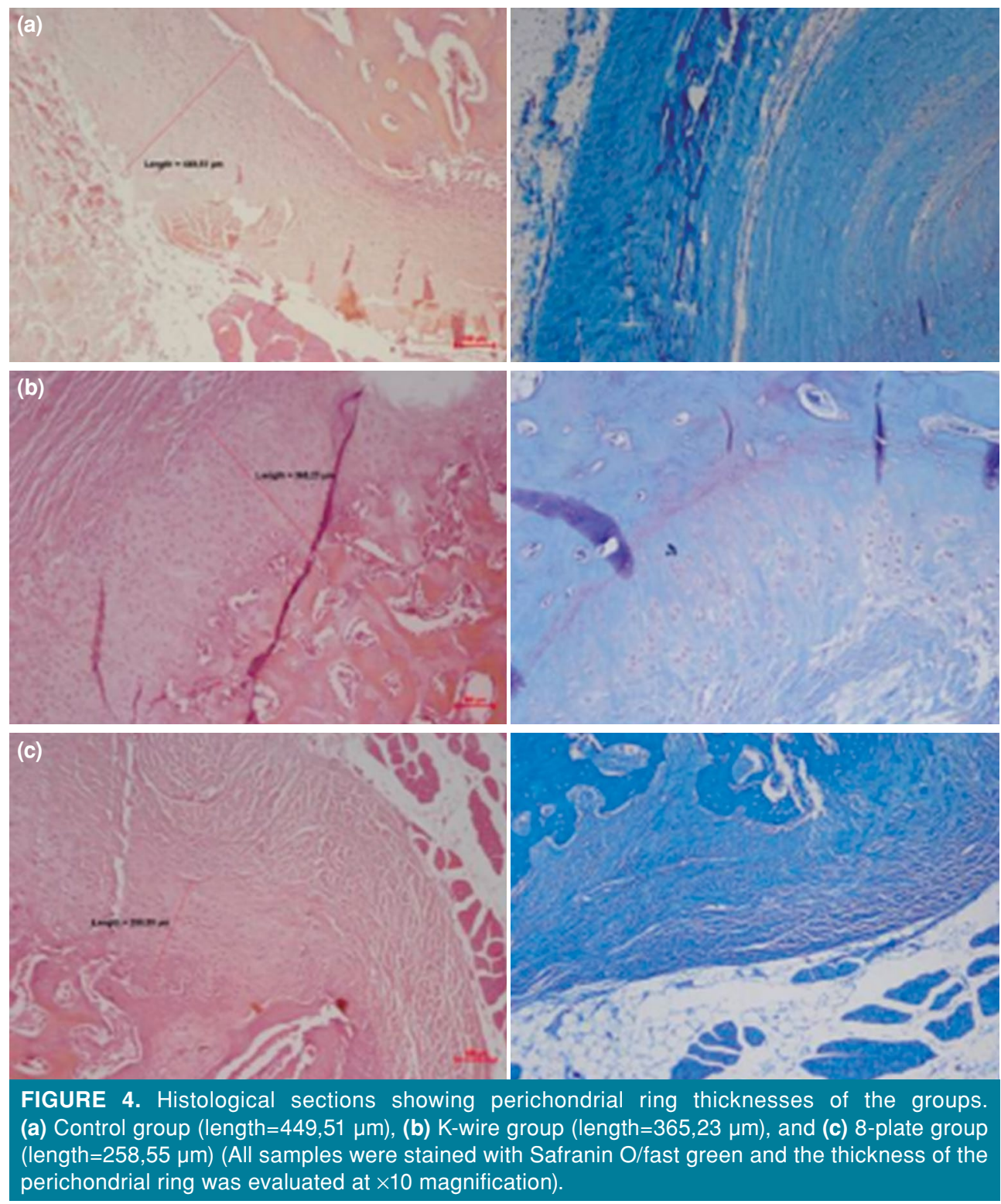

comparison of more than two groups of quantitative variables that did not show normal distribution. A $p$ value of $<0.05$ was considered statistically significant.

\section{RESULTS}

No statistically significant difference was observed in terms of ALDA, TSA, and MSA in the early postoperative radiographs among the groups $(\mathrm{p}=0.630, \mathrm{p}=0.751$, and $\mathrm{p}=0.673$; respectively) (Table I). However, in both K-wire and 8-plate groups, the early postoperative ALDA and TSA were greater than the sacrification ALDA and TSA $(p=0.028$ and $\mathrm{p}<0.001$, respectively). The early postoperative MSA was lower than the sacrification MSA in both
K-wire and 8-plate groups $(\mathrm{p}<0.001)$. A statistically significant difference was observed between groups in terms of sacrification ALDA, TSA, and MSA values $(p<0.05)$. As a result of Dunn-Bonferroni post-hoc tests, the ALDA value in the control group was higher than the K-wire and 8-plate groups $(p=0.005$ and $p=0.001$; respectively), while the TSA value in the $\mathrm{K}$-wire group was lower than the control and 8-plate groups ( $\mathrm{p}=0.001$ and $\mathrm{p}=0.016$; respectively) and the MSA value in the control group was lower than the $\mathrm{K}$-wire and 8-plate groups $(\mathrm{p}<0.001$ and $\mathrm{p}=0.009$; respectively). In the 8 -plate group, no statistically significant results were obtained in angle changes between the screws (Table II). 


\begin{tabular}{|c|c|c|c|c|c|c|c|}
\hline \multicolumn{8}{|c|}{$\begin{array}{c}\text { TABLE I } \\
\text { Taluatio }\end{array}$} \\
\hline & \multicolumn{2}{|c|}{ K-wire } & \multicolumn{2}{|c|}{ Control } & \multicolumn{2}{|c|}{ 8-Plate } & \multirow[b]{2}{*}{$p \dagger$} \\
\hline & Mean & Min-Max & Mean & Min-Max & Mean & Min-Max & \\
\hline \multicolumn{8}{|l|}{ ALDA } \\
\hline Early postoperative & 92.3 & $91.45-92.7$ & 92.1 & $91.8-92.65$ & 92.6 & $91-93.95$ & $0.630^{\star}$ \\
\hline Sacrification & 86.45 & $85.65-87$ & 93.35 & $92.8-93.65$ & 84.05 & $81.4-87.75$ & $<0.001^{* *}$ \\
\hline$p \ddagger$ & \multicolumn{2}{|c|}{$<0.001^{* *}$} & \multicolumn{2}{|c|}{$0.028^{*}$} & \multicolumn{2}{|c|}{$<0.001^{* *}$} & \\
\hline \multicolumn{8}{|l|}{ TSA } \\
\hline Early postoperative & 88.8 & $88.1-88.95$ & 88.9 & $88.45-89.35$ & 88.75 & $88.05-89.4$ & $0.751^{\star *}$ \\
\hline Sacrification & 78.15 & $77.7-79.3$ & 86.8 & $86.1-87.15$ & 81.85 & $81.55-83.35$ & $0.001^{* *}$ \\
\hline$p \ddagger$ & \multicolumn{2}{|c|}{$<0.001^{* *}$} & \multicolumn{2}{|c|}{$<0.001^{* *}$} & \multicolumn{2}{|c|}{$<0.001^{\star *}$} & \\
\hline \multicolumn{8}{|l|}{ MSA } \\
\hline Early postoperative & 7 & $6.75-7.4$ & 6.95 & $6.4-7.45$ & 7.01 & $6.65-8.3$ & $0.673^{* *}$ \\
\hline Sacrification & 14.05 & $13.6-14.65$ & 7 & $6.85-7.25$ & 13.55 & $12.7-14.45$ & $<0.001^{\star *}$ \\
\hline$p \ddagger$ & \multicolumn{2}{|c|}{$<0.001^{* *}$} & \multicolumn{2}{|c|}{0.721} & \multicolumn{2}{|c|}{$<0.001^{* *}$} & \\
\hline
\end{tabular}

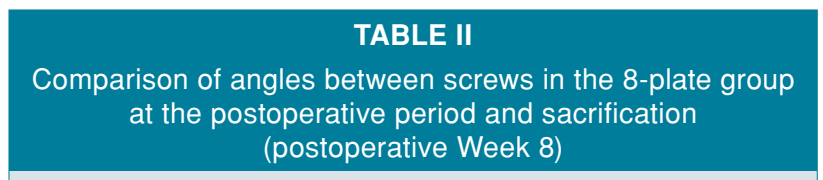

\begin{tabular}{lcc} 
& \multicolumn{2}{c}{ Angle between screws } \\
\cline { 2 - 3 } & Mean & Min-Max \\
\hline Early postoperative & 8 & $5.75-12.3$ \\
Sacrification & 5.3 & $2.9-10$ \\
Total & 7.1 & $4-11.35$ \\
$p^{*}$ & \multicolumn{2}{c}{0.161} \\
\hline
\end{tabular}

* Mann-Whitney U test, median value was presented as first quarter, third quarter.

In histological evaluation, there was a statistically significant difference between the groups in terms of perichondral ring thickness $(\mathrm{p}<0.001)$. As a result of Dunn-Bonferroni post-hoc tests, the histological cross-section value of the control group was greater than the K-wire and 8-plate groups $(\mathrm{p}<0.001)$. The histological cross-section value of the K-wire group was greater than the 8-plate group $(\mathrm{p}<0.001)$ (Table III). A 10-fold price difference was found between the K-wire and cerclage used in Group 1 and the cost of the 8-plate used in Group 2 (\$41 and $\$ 395$, respectively).

\section{DISCUSSION}

The main findings of the present study are that both of the K-wire and 8-plate groups show similar

\begin{tabular}{|c|c|c|}
\hline \multicolumn{3}{|c|}{$\begin{array}{l}\text { Comparison of groups in terms of perichondral ring } \\
\text { thickness in histological evaluation }\end{array}$} \\
\hline & \multicolumn{2}{|c|}{ Perichondrial ring thickness } \\
\hline & Mean & Min-Max \\
\hline Control & 450.71 & $445.76-477.68$ \\
\hline 8-Plate & 258.55 & $246.65-261.78$ \\
\hline K-wire & 366.78 & $364.41-372.63$ \\
\hline Total & 366.78 & $261.78-477.68$ \\
\hline$p^{*}$ & \multicolumn{2}{|c|}{$<0.001^{\star *}$} \\
\hline
\end{tabular}

angulation effects in the proximal tibial zone and histologically less damage to the perichondral ring in the K-wire group, compared to the 8-plate group. To the best of our knowledge, the current study is the first experimental animal study in the literature to compare K-wire and 8-plate in epiphysiodesis papers.

Tension band technique with K-wire and cerclage application is frequently used in orthopedic practice as a method that can be applied in many areas such as olecranon and medial malleolar fractures, providing pressure to the fracture line and being cheaper than osteosynthesis with plate and screws. ${ }^{[10]}$ It has been shown in the previous literature that physeal growth can be kept under control by creating pressure on the physical line. ${ }^{[11]}$ In our study, the reason for performing the tension band technique by using 
K-wire and cerclage is to keep the growth under control by applying pressure on the physeal plate, to reduce the pressure of the perichondrial ring formed by the wide surface of the plate, and to use a more cost-effective method. The 8-plate application is used in daily orthopedic practice to prevent deformities and curves in the limb. ${ }^{[8]}$ However, an animal experiment study in a pig model showed that 8-plate might cause perichondral ring damage by creating pressure on the chondral ring underneath. ${ }^{[9]}$ The present study showed that both the K-wire and the 8-plate reduced perichondral ring thickness in histological evaluation, compared to the control group. However, the reduction of the perichondral ring thickness in the K-wire group was less than the damage caused by the 8-plate. We believe that this depends on the pressure created by the cerclage wires being less than 8-plate and the cerclage profile is thinner than the 8-plate.

In a study, Schroerlucke et al. ${ }^{[13]}$ reported that implant failure due to screw breakage in the tibia and reoperation might be required in Blount patients and rickets with 8-plate treatment. In the present study, implant failure was not observed in any group. In revision surgeries due to implant failure, the risk of perichondral ring injury increases, probably leading to permanent damage. ${ }^{[12,19]}$ The experimental study of $\mathrm{Wu}$ et al. ${ }^{[9]}$ showed that the pressure in the perichondrial ring increased and the perichondrial ring thickness decreased after epiphysiodesis with 8-plate to the pig tibia. The tension band techniques provide temporary correction, when the curve improves the implants are removed and the limb continues to grow up ${ }^{[12,13]}$ The perichondrial ring can be damaged by implant pressure or during implant removal surgery ${ }^{[1,7,20]}$ Damage to the perichondrial ring can cause bar formation in the growth plate, thereby leading to permanent physeal damage and angular deformities. ${ }^{[20]}$ The K-wires and cerclage may be an alternative method to minimize this effect. In the current study, the osseous bar formation was not observed in the histological examination.

The angular changes in the limb after deformity correction surgeries and epiphysiodesis can be measured radiographically. ${ }^{[21,22]}$ The ALDA, MSA, and TSA are frequently used radiological measurements for proximal tibial deformities. ${ }^{[7,9,21,22]}$ The alteration of ALDA and MSA is important for coronal plane deformities, while the alteration of TSA is important for sagittal plane deformities ${ }^{[7]}$ In our study, the ALDA and MSA angles were similar between K-wire and 8-plate groups. Due to the compressive forces of 8-plate and $\mathrm{K}$-wires and cerclage, proximal tibial angulation may occur. In this study, both groups showed similar effects; as such, the ALDA rates were lower than the control group and MSA rates were higher than the control group. However, the TSA was lower in the K-wire group. We believe that the compressive power of two proximal K-wires that placed in a $5 \mathrm{~mm}$ interval provides the lower TSA in the K-wire group. Besides, those K-wires apply the compressive power on the proximal articular surface of the tibia significantly at two points. Although three staples are used in the Blount's staple technique, some authors have advocated that two nails are sufficient with $U$ nails made of stiff material. ${ }^{[23-25]}$ Staple configuration changes compressive forces in different axes and this can affect coronal and sagittal plane angulation. We also consider that the distance between two K-wires is different than the width of the plate, making it more effective on the lateral angle alteration.

Review of the literature reveals that the different angles of the screws applied with the 8-plate do not considerably affect the results. ${ }^{[26]}$ In our study, screws were applied parallel to each other in the 8-plate group. There was no statistically significant difference in the angle change between screws. We believe that this change is not significant due to the short follow-up period and the relatively small rabbit tibia. Tension band fixation used for fracture compression in orthopedic trauma surgery was preferred to provide epiphysiodesis in the present study. ${ }^{[15,16]}$ We found that the tension band technique performed with $\mathrm{K}$ wire and cerclage could control the growth in the epiphysis by the aid of the compression created in the physeal growth plate of the subjects and was as effective as 8-plate. One of the hypotheses of the present study was to reduce the cost of the implants used in surgical procedures. The average implant cost in the K-wire group was approximately 10 times lower than the 8-plate group ( $\$ 41$ and $\$ 395$, respectively). Burghart et al. ${ }^{[19]}$ reported that the cost of U-nail was lower than 8-plate. The effective results over of $90 \%$ were previously achieved with the extraperiosteal implants during implant fixation and removal. ${ }^{[27]}$ In our study, no follow-up was performed after implant removal. However, it should be remembered that complications such as rebound effect and implant failure can be seen in the long-term. ${ }^{[2]}$ Implant failure is more common in patients with obesity and the population under the age of 10 years, and it is recommended to use larger or more solid screws with stronger constructs. ${ }^{[28]}$ Different sizes of K-wire and cerclage are used frequently in the clinical practice. We believe that a larger K-wire and cerclage can be used to increase the fixation strength. 
The main limitations of the present study are that the epiphysiodesis was performed in an animal model, the follow-up period was shorter than the hemiepiphysiodesis techniques applied in the clinic, and there was no follow-up after implant removal. Besides, to apply the technique in daily orthopedic practice, a more detailed information is needed in animals with larger epiphysis, after longer follow-up and by evaluating the growth of the epiphyseal plate after implant removal.

In conclusion, both of the K-wire and 8-plate groups showed similar angulation effects in the proximal tibia, although histologically less damage to the perichondrial ring was observed in the K-wire group, compared to the 8-plate group. Although this study reveals that the tension band technique is as successful as fixation with 8-plate in terms of tibial proximal hemiepiphysiodesis and causes less perichondral damage, the results seem to have limited validity and effectivity to be used in clinical cases.

\section{Declaration of conflicting interests}

The authors declared no conflicts of interest with respect to the authorship and/or publication of this article.

\section{Funding}

Turkish Republic, University of Health Sciences, Scientific Research Projects Unit funds were received in support of this work (2018/010).

\section{REFERENCES}

1. Yu Y, Rodriguez-Fontan F, Eckstein K, Muralidharan A, Uzcategui AC, Fuchs JR, et al. Rabbit model of physeal injury for the evaluation of regenerative medicine approaches. Tissue Eng Part C Methods 2019;25:701-10.

2. Sabharwal S, Sabharwal S. Growth plate injuries of the lower extremity: Case examples and lessons learned. Indian J Orthop 2018;52:462-9.

3. Rodrigues NVM, Guarniero R, Boas PJFV, de Miranda BR, Montenegro NB. Hemiepiphysiodesis using eight-plate versus Blount staple to correct genu valgum and genu varum. Acta Ortop Bras 2020;28:195-8.

4. Oto M, Yilmaz G, Bowen JR, Thacker M, Kruse R. Adolescent Blount disease in obese children treated by eight-plate hemiepiphysiodesis. Eklem Hastalik Cerrahisi 2012;23:20-4.

5. Blount WP. A mature look at epiphyseal stapling. Clin Orthop Relat Res 1971;77:158-63.

6. Zajonz D, Schumann E, Wojan M, Kübler FB, Josten C, Bühligen $U$, et al. Treatment of genu valgum in children by means of temporary hemiepiphysiodesis using eight-plates: Short-term findings. BMC Musculoskelet Disord 2017;18:456.

7. Aykut US, Yazici M, Kandemir U, Gedikoglu G, Aksoy MC, Cil A, et al. The effect of temporary hemiepiphyseal stapling on the growth plate: A radiologic and immunohistochemical study in rabbits. J Pediatr Orthop 2005;25:336-41.

8. Stevens PM. Guided growth for angular correction: A preliminary series using a tension band plate. J Pediatr Orthop 2007;27:253-9.
9. Wu Z, Zhao D, Zhao L, Liu J, Li H, Zhu J, et al. A comparison between a hinged plate and screw system and a conventional tension-band plate and screw system used for correction of an angular deformity of the lower limb: An animal study. J Orthop Surg Res 2015;10:57.

10. Bosman WPF, Emmink BL, Bhashyam AR, Houwert RM, Keizer J. Intramedullary screw fixation for simple displaced olecranon fractures. Eur J Trauma Emerg Surg 2020;46:83-9.

11. Son SM, Park IH, Oh CW, Lee HJ, Park BC, Choi JY. A histomorphometric study of cellular layers after hemiepiphyseal stapling on the physeal plate in rabbits. J Orthop Sci 2013;18:152-8.

12. Castañeda P, Urquhart B, Sullivan E, Haynes RJ. Hemiepiphysiodesis for the correction of angular deformity about the knee. J Pediatr Orthop 2008;28:188-91.

13. Schroerlucke S, Bertrand S, Clapp J, Bundy J, Gregg FO. Failure of Orthofix eight-plate for the treatment of Blount disease. J Pediatr Orthop 2009;29:57-60.

14. Ding J, Jin F, Zhao X, Zhao L, Wu Z, Li J. Hemiepiphysiodesis stapling induces ER stress apoptosis and autophagy in rat growth plates. Am J Transl Res 2019;11:1486-97.

15. Nowotny J, Bischoff F, Ahlfeld T, Goronzy J, Tille E, Nimtschke $U$, et al. Biomechanical comparison of biand tricortical k-wire fixation in tension band wiring osteosynthesis. Eur J Med Res 2019;24:33.

16. Kuru T, Akpınar F, Işık C, Özkılıç R, İpek S, Mutlu $\dot{I}$, et al. Biomechanical comparison of a new handy tension band with malleolar screw, bicortical screw and conventional tension band for the fixation of transverse medial malleolar fractures. Eklem Hastalik Cerrahisi 2019;30:301-8.

17. Tolentino ES, Yamashita FC, de Albuquerque S, Walewski LA, Iwaki LCV, Takeshita WM, et al. Reliability and accuracy of linear measurements in cone-beam computed tomography using different software programs and voxel sizes. J Conserv Dent 2018;21:607-12.

18. Corominas-Frances L, Sanpera I, Saus-Sarrias C, TejadaGavela S, Sanpera-Iglesias J, Frontera-Juan G. Rebound growth after hemiepiphysiodesis: An animal-based experimental study of incidence and chronology. Bone Joint J 2015;97-B:862-8.

19. Burghardt RD, Herzenberg JE, Andre Strahl, Bernius P, Kazim MA. Treatment failures and complications in patients with Blount disease treated with temporary hemiepiphysiodesis: a critical systematic literature review. J Pediatr Orthop B 2018;27:522-9.

20. Burghardt RD, Specht SC, Herzenberg JE. Mechanical failures of eight-plateguided growth system for temporary hemiepiphysiodesis. J Pediatr Orthop 2010;30:594-7.

21. Burghardt RD, Herzenberg JE. Temporary hemiepiphysiodesis with the eight-plate for angular deformities: Mid-term results. J Orthop Sci 2010;15:699704 .

22. Kulkarni RM, Ilyas Rushnaiwala FM, Kulkarni GS, Negandhi R, Kulkarni MG, Kulkarni SG. Correction of coronal plane deformities around the knee using a tension band plate in children younger than 10 years. Indian J Orthop 2015;49:208-18.

23. Raab P, Wild A, Seller K, Krauspe R. Correction of length discrepancies and angular deformities of the leg by Blount's epiphyseal stapling. Eur J Pediatr 2001;160:668-74. 
24. Gorman TM, Vanderwerff R, Pond M, MacWilliams B, Santora SD. Mechanical axis following staple epiphysiodesis for limb-length inequality. J Bone Joint Surg [Am] 2009;91:2430-9.

25. Hillebrand H, Sattelberger J, Gosheger G, Frommer A, Moller-Madsen B, Rahbek O, et al. Comparison of temporary epiphysiodesis with RigidTacks ${ }^{\mathrm{TM}}$ and Blount-Staples in a porcine animal model using magnetic resonance imaging. J Orthop Res 2020;38:946-53.
26. Eltayeby HH, Iobst CA, Herzenberg JE. Hemiepiphysiodesis using tension band plates: Does the initial screw angle influence the rate of correction? J Child Orthop 2019;13:62-6.

27. Mielke $\mathrm{CH}$, Stevens PM. Hemiepiphyseal stapling for knee deformities in children younger than 10 years: A preliminary report. J Pediatr Orthop 1996;16:423-9.

28. Fan B, Zhao C, Sabharwal S. Risk factors for failure of temporary hemiepiphysiodesis in Blount disease: A systematic review. J Pediatr Orthop B 2020;29:65-72. 